\title{
Viewpoint and orientation influence picture recognition in the blind
}

\author{
Morton A Heller, John M Kennedy $\uparrow$, Ashley Clark, Melissa McCarthy, Amber Borgert, \\ Lindsay Wemple, Erin Fulkerson, Nicole Kaffel, Amy Duncan, Tara Riddle \\ Department of Psychology, Eastern Illinois University, 600 Lincoln Avenue, Physical Sciences Building, \\ Charleston, IL 61920, USA; e-mail: maheller@eiu.edu; Division of Life Sciences, University of Toronto \\ at Scarborough, 1265 Military Trail, Toronto, Ontario M1C 14A, Canada \\ Received 27 April 2005, in revised form 8 November 2005; published online 13 October 2006
}

\begin{abstract}
In the first three experiments, subjects felt solid geometrical forms and matched raised-line pictures to the objects. Performance was best in experiment 1 for top views, with shorter response latencies than for side views, front views, or 3-D views with foreshortening. In a second experiment with blind participants, matching accuracy was not significantly affected by prior visual experience, but speed advantages were found for top views, with 3-D views also yielding better matching accuracy than side views. There were no performance advantages for pictures of objects with a constant cross section in the vertical axis. The early-blind participants had lower performance for side and frontal views. The objects were rotated to oblique orientations in experiment 3. Early-blind subjects performed worse than the other subjects given object rotation. Visual experience with pictures of objects at many angles could facilitate identification at oblique orientations. In experiment 5 with blindfolded sighted subjects, tangible pictures were used as targets and as choices. The results yielded superior overall performance for 3-D views (mean, $M=74 \%$ correct) and much lower matching accuracy for top views as targets ( $M=58 \%$ correct). Performance was highest when the target and matching viewpoint were identical, but 3-D views ( $M=96 \%$ correct) were still far better than top views. The accuracy advantage of the top views also disappeared when more complex objects were tested in experiment 6. Alternative theoretical implications of the results are discussed.
\end{abstract}

\section{Introduction}

A considerable body of recent research suggests that tangible pictures may hold considerable utility for blind persons, and we are just beginning to discover the variables that matter (Heller and Ballesteros 2006; Kennedy 2000; Millar 1994). Haptic texture gradients can provide pictorial information (Holmes et al 1998; Thompson and Chronicle 2002; Thompson et al 2003), for example. The viewpoint of the picture may be important in drawings of cars, insects, and people (Heller 2002; Heller et al 2002; Kennedy 1993). There is a large literature on the question whether object recognition is viewpoint-dependent in vision, but less is known about this in touch (see Biederman 2001; Biederman and Gerhardstein 1993; Tarr and Bülthoff 1995; Tarr et al 1998). While we have some information about what may constitute a canonical or familiar view in vision, this knowledge is not available for touch. Many blind people may have had little or no experience with tangible pictures, but, of course, there are some exceptions (see Kennedy and Juricevic 2006).

Heller et al (2002) reported that sighted and blind subjects performed far better with top views of geometrical solids than they did with frontal views or 3-D views with overlap, foreshortening, and other perspective features. All groups of subjects were much faster with the top views, and much slower when attempting to match a solid object with picture choices with 3-D features. It was notable that all groups of blind subjects were much faster than the blindfolded sighted subjects. The late-blind (LB) and very-low-vision (VLV) subjects were much more accurate than the blindfolded sighted (BS) subjects, while maintaining their advantage in speed over the sighted. Early-blind (EB) subjects were as accurate as the BS subjects, but were much faster. 
The Heller et al (2002) results are inconsistent with theories contending that visual experience is necessary for the interpretation of space or tangible pictures (Revesz 1950). Indeed, the congenitally blind (CB) and BS subjects had similar levels of accuracy. However, there are many ways a tangible picture might depict depth relations, and offer problems for CB subjects (Heller et al 1996; Holmes et al 1998). Linear perspective, for example, uses convergence, and Eriksson (1998, pages 153-155) suggests haptic pictures in parallel perspective would be more readily understood. Kennedy (2003) and Heller and Kennedy (1990) noted that haptic pictures drawn by some blind adults have used perspective features related to the observer's vantage point, but there is no single privileged viewpoint in touch as there is in vision. Close vantage points would yield convergence in linear perspective. Some CB persons may be unfamiliar with the rules governing the depiction of linear perspective, and this could present problems for their interpretation of 3-D tangible drawings: 3-D drawings involve perspective, and this is intimately linked to viewpoint.

As an alternative to linear and parallel perspective, some pictures might be taken to be cross sections. All the Heller et al (2002) groups had lower accuracy with pictures of a pyramid. A pyramid varies more in its cross section than objects such as a block. Consequently in the present study we explicitly manipulated the cross section of stimulus standard objects, with half of them having constant cross sections and the other half variable cross sections. Given the results of earlier research, it was expected that the subjects would have greater difficulty with objects with variable cross sections in the vertical dimension.

In experiment 3 of the present study we also manipulated the orientation of the objects. This was done to increase task difficulty. Blind subjects performed better than the sighted when coping with Braille that was left-right reversed, but not with Braille rotated $\pm 45^{\circ}$ from the upright (Heller et al 1999). Blind subjects were able to cope with rotation of number patterns printed on their palms (Heller 1989). Compared to the sighted, CB subjects probably have less everyday experience with tasks that demand mental rotation of objects or patterns.

The present study is relevant to the issues of viewpoint-dependence in touch. Newell et al (2002) have reported that, in touch, the backs of objects are understood more readily than fronts, likely because the hand's posture is more comfortable and flexible than when pressing from the front. Furthermore, small objects can be felt on all sides at once. However, Newell et al did not study picture perception, with its exploration from above, and its standard schemas. Also, Newell et al's results may be specific to the kinds of Lego-block 3-D solids in their experiments. Further, we do not presently know whether some vantage points are strongly or weakly privileged for visually impaired subjects who have had a minimal or no experience with tangible 2-D drawings.

If visual experience is valuable for understanding tangible pictures of solids, then EB subjects might be expected to show lower levels of performance than BS subjects. However, if, in principle, outline drawings depicting edges of objects are as meaningful for touch as for sight (see Heller et al 1996, 2002; Kennedy 1993), then EB participants are predicted to fare well with line drawings of standard objects, projected in a favored manner to a suitable vantage point. This prediction is bolstered by imagery, and its vantage points, having effects that can be obtained from the blind as well as the sighted (Kerr 1983; Marmor and Zaback 1976; Prather and Sathian 2002; Zimler and Keenan 1983).

In the first experiment reported here we manipulated the vantage point of the picture and cross section of the solid (either variable or constant). Since tactile pictures are explored from above the picture, we expected that BS subjects would perform best with top views, and less well with frontal or side views. Also, stimuli for which linear projection, parallel projection, and cross sections are identical were expected to be 
identified most readily. Stimuli that varied in cross section would be harder, for two reasons. First, fewer projections produced the form. Lines are generally taken to be the occluding edges of objects, not a cross section which cuts through a continuous surface. To be complete, we mention that the effect of viewpoint might depend upon the specific stimuli and cross section.

\section{Experiment 1: Viewpoint effects in blindfolded sighted (BS) subjects}

\subsection{Method}

2.1.1 Design. The experiment was a repeated-measures design, with the variables being viewpoint (top, front, 3-D, and left side view) and cross section of the objects (constant or variable). Objects were considered in the analyses of latency scores. The order of presentation of the viewpoints was randomized, but blocked by viewpoint.

2.1.2 Participants. There were sixteen undergraduate volunteers (nine females, seven males). Fourteen were right-handed, one was left-handed, and one was ambidextrous. They received course credit for their participation.

2.1.3 Stimuli and apparatus. The stimuli were solid wooden shapes machined from hard maple, sanded smooth, and finished with a polyurethane varnish (see figure 1). The objects were fixed in place during the experiment, and could not be moved. The wooden objects were approximately $8.9 \mathrm{~cm}$ tall by $7.5 \mathrm{~cm}$ wide (the 'egg' was $8.4 \mathrm{~cm}$ tall by $5 \mathrm{~cm}$ wide, with a flat base). Some of the objects were truncated by a plane at $45^{\circ}$, tilted top-far and bottom-near with respect to the subject's vantage point. Drawings were to scale and nearly life-sized. The frontal viewpoint was drawn so that the bottom of the object was at eye-height at a distance of $50 \mathrm{~cm}$ from the observer, with a vertical picture plane. That is, bottom edges were drawn with horizontal lines, but linear perspective required foreshortening of side faces, use of obliques for receding edges, and use of curves for ovals seen from the side. The side view (from the left) was drawn from a similar vantage point. The top view was drawn as if looking straight down on the top of the object from a height of $50 \mathrm{~cm}$ (and could be in parallel or linear perspective, or be a cross section). The 3-D views were linear-perspective depictions from a vantage point of $30 \mathrm{~cm}$ above the top front edge and $50 \mathrm{~cm}$ away. The drawings were produced by monocularly viewing the objects through a frontally placed clear acrylic panel that was abutted to the front of a light table. The objects were placed immediately behind the acrylic panel on the surface of the light table, and clear tracing material was placed upon the drawing surface of the acrylic. The images were viewed monocularly at the distances previously mentioned. The raised-line drawings in experiments 1-3 were produced on swell-paper, a paper that produces a raised line when heated and cooled, and mounted on thick matte board.

The objects with identical cross sections through their widest extents in a vertical frontal plane were the cylinder, rectangular parallelepiped, and vertically sectioned cylinder; the other objects had different vertical cross sections (see figure 1). These cross sections are identical to occluding boundaries of the objects' surfaces, since they are through the widest extents of the objects. They differed from the outline drawings from a vantage point in that outline drawings include features on surfaces facing the vantage point. (A line drawing of a cross section through one's head has an empty interior, but an outline drawing includes nose, mouth, and eyes). Top views in figure 1 are cross sections in the horizontal plane, and show surface boundaries. Only two of the four objects in figure 2 were truncated, since the aim of the experiment was to study the effect of variability in the cross section along the vertical axis. To that aim, half of the objects varied in cross section, but the others did not. The egg already varied in cross section in the vertical axis, so it was not truncated. Note that canonical views for vision were not selected. This would have biased the task toward vision, and 
rectangular parallelepiped

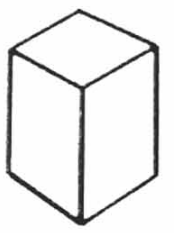

cylinder

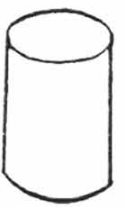

vertically sectioned cylinder

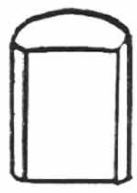

egg

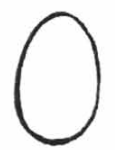

truncated rectangular parallelepiped

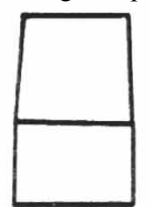

truncated cylinder

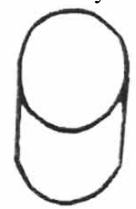

(a)
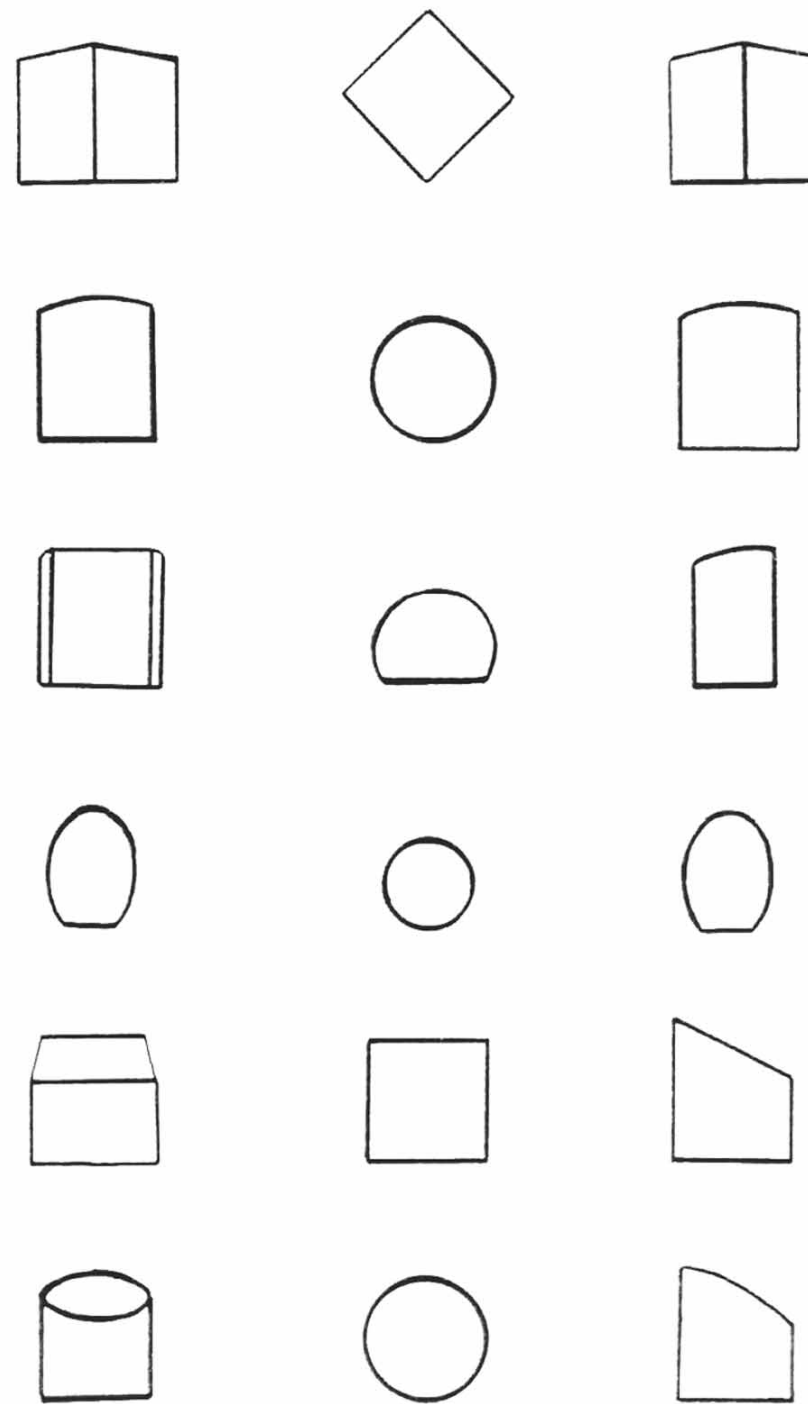

(b)

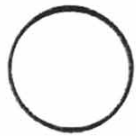

(c)
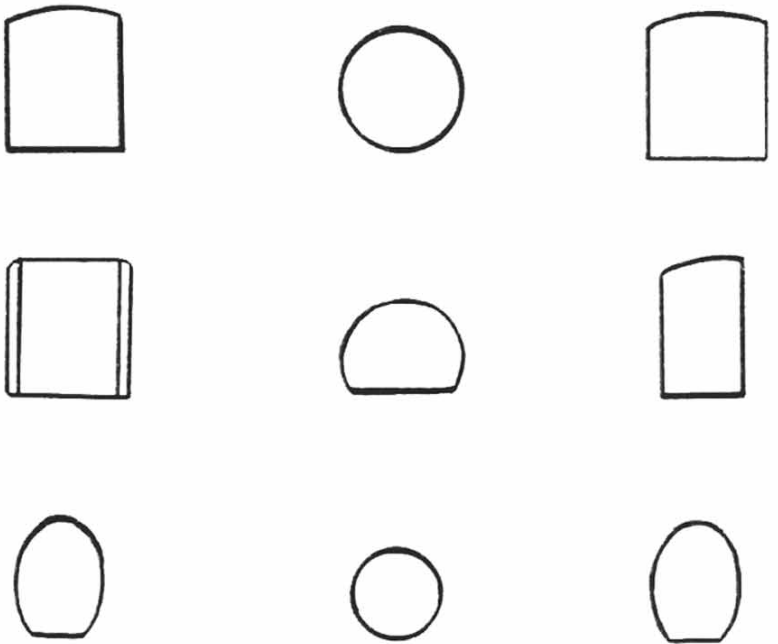

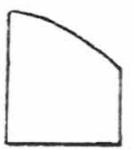

(d)

Figure 1. Drawings used in experiments 1-4: (a) the 3-D view, (b) the frontal view, (c) top views, and (d) the left side view.

would not have provided for a 'fair' comparison in the second experiment reported here with blind participants. This was an attempt to control for the relative lack of familiarity of blind people with pictures of geometrical forms.

Subjects responded by choosing a picture from a choice set. The set consisted of the correct choice and three other randomly selected pictures of the other objects, but from the same viewpoint. The only exception to this involved instances where random selection yielded identical drawings of the incorrect choice and correct choice (eg the top views of the cylinder and truncated cylinder were both equivalent circles); here an alternative picture choice was randomly selected from the same viewpoint. The objects were placed at the top of the framework designed to hold the stimuli flat on the table, and the picture choices were placed closer to the subjects (see figure 3). 

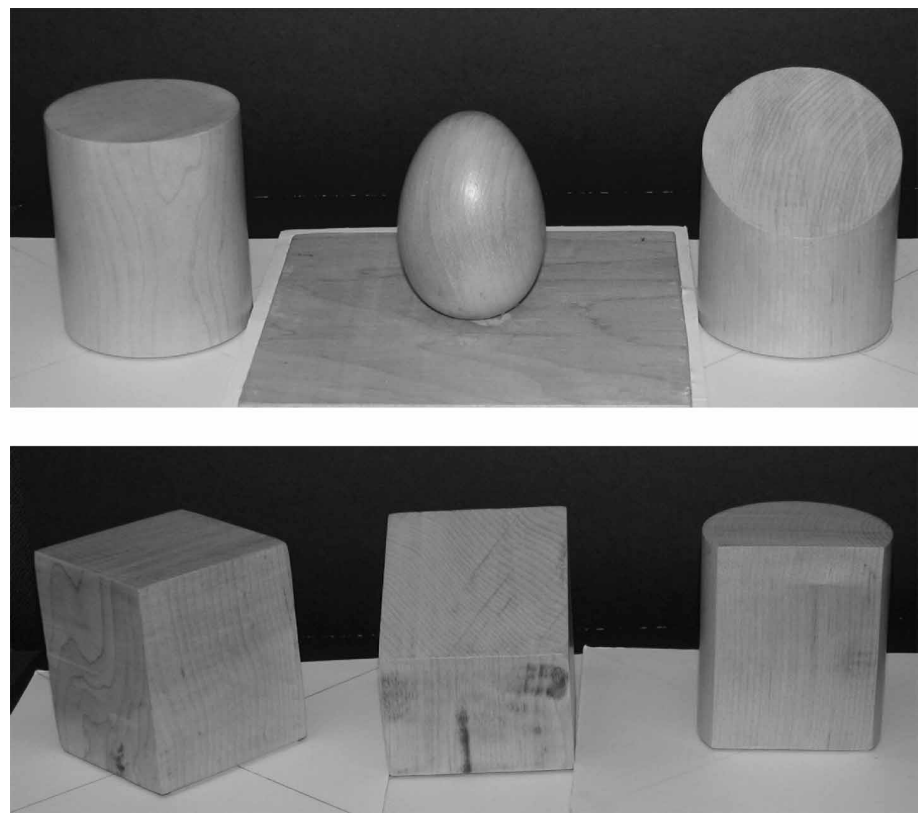

Figure 2. Wooden objects used in experiments $1-3$.

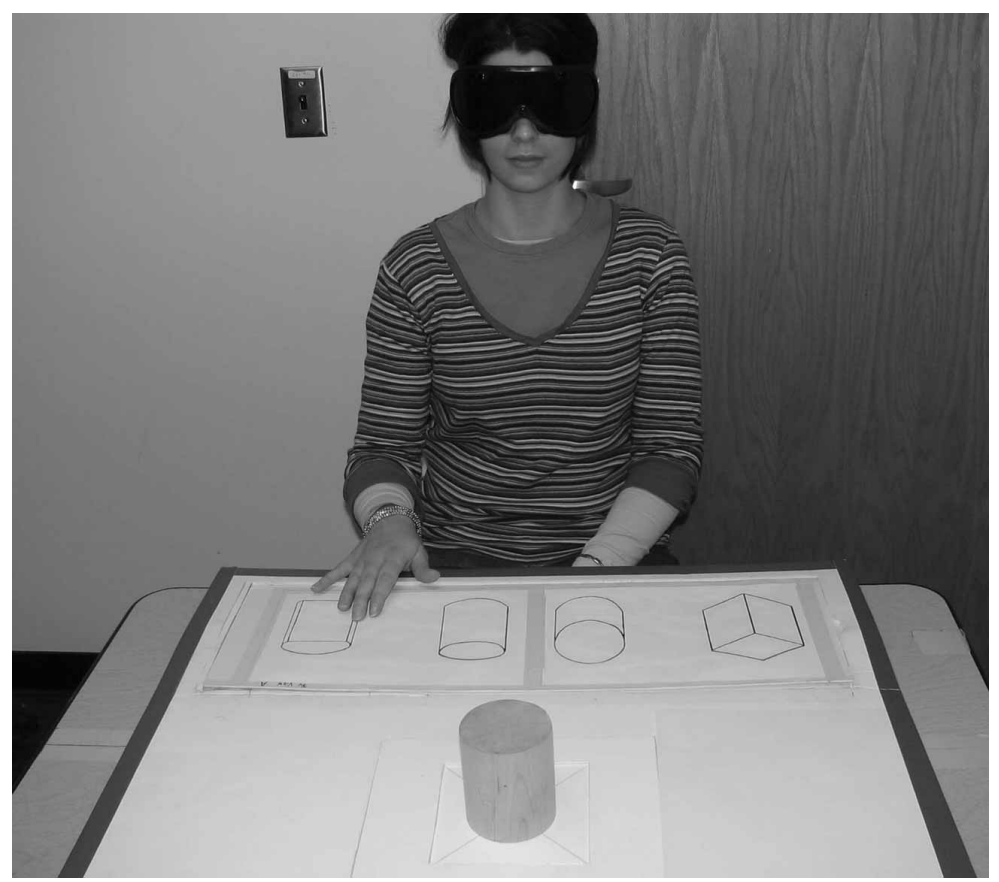

Figure 3. The experimental setup, with EF at the table. The framework for holding the objects and picture choices can be seen, along with a target object and set of choices.

As previously mentioned, the objects were fixed in place and could not be moved in this and subsequent experiments reported here. The space between the four picture choices was approximately equal.

2.1.4 Procedure. Participants were given a standard wooden object and four picture choices. They were told to feel the immobile standard object, and then feel the four choices 
from left to right. After feeling all four choices, they were to tap the picture that showed the standard object. Subjects were told that one of the choices was correct, but that the other choices were drawings of the other objects used as models for the pictures. Time limits were not imposed, but subjects were told that they would be timed with a stopwatch from the moment that they felt the standard wooden object until they tapped their choice. They were instructed to try for accuracy, and take as much time as they needed to get their answers correct. The subjects were allowed to use one or both hands to feel the objects and the picture choices. They were told that they could go back and check the wooden object whenever they wished. Thus, if they desired, subjects could simultaneously feel the standard object with one hand and the pictures with the other. This procedure was followed to insure that performance was not dependent on memory. Feedback on performance was not given.

The subjects were given prior information about the viewpoint of the picture choices before the beginning of each block of trials. For the frontal views, the subjects were told that the objects were drawn from the front, with the bottom of the object at eye-height. For the 3-D views, the subjects were told that the objects were depicted as if they could see them from where they were sitting if they were to look down at them at an angle. They were also told that the 3-D views showed the tops of the objects and their fronts at the same time, and would look like they would appear if they were not blindfolded. The instructions for the top views indicated that the drawings were from the vantage point of directly above, as in a 'bird's-eye-view'. The left-side-view instructions emphasized that the pictures showed the object from the left, with the bottom of the object at eye-height while they were drawn. In addition, the experimenter took the subjects' preferred hands and moved them from their eyes to the object. This was designed to clarify the viewpoint. For example, for the left side views, the subject's left hand was moved to the left side of a sample object while a verbal explanation was given. The sample object was a triangular prism, and was not one of the target stimuli in the experiments reported here. For the frontal views, the subject's preferred hand was moved from his/her eyes straight toward the sample object. Similar concrete demonstrations were used for the 3-D views and the top views.

\subsection{Results and discussion}

The results of experiment 1 are shown in tables 1 and 2. Subjects performed much faster on the top views (mean, $M=38.12 \mathrm{~s}$ ) than the other views. An ANOVA on mean time scores as a function of viewpoint of the pictures and cross section of the objects showed that the effect of viewpoint was highly significant $\left(F_{3,45}=12.60, p<0.001\right)$. The main effect of the cross section of the objects failed to reach significance $\left(F_{1,15}=2.4, p=0.14\right)$, but the interaction between viewpoint of the pictures and cross section of the objects was highly significant $\left(F_{3,45}=3.61, p<0.025\right)$. The simple effect of cross section was significant only for the 3 -D views $\left(F_{1,15}=8.19, p=0.012\right)$. Cross section did not alter response latencies for the other viewpoints (all $p \mathrm{~s}>0.20$ ). Subjects were faster with the objects of variable cross section and 3-D pictures than 3-D pictures of the objects with a constant cross section.

The analyses of accuracy scores indicate better performance for the top views, at $83.3 \%$ correct, with lower accuracy for the side views $(67.8 \%$ correct $)$. A mixed-factor ANOVA on number correct showed that the main effect of viewpoint failed to reach significance $\left(F_{3,45}=2.24, p=0.097\right)$. The effect of cross section was marginally significant $\left(F_{1,15}=4.44, p=0.052\right)$. The interaction between viewpoint and cross section of the objects was nonsignificant $\left(F_{3,45}=0.8, p=0.51\right)$. Counter to the original predictions, the subjects did slightly better with the objects with variable cross sections than those with constant cross sections. 
Table 1. Mean number correct and mean time per object (seconds) in experiment 1 as a function of cross section of the objects and viewpoint of the haptic pictures (with standard deviations in parentheses).

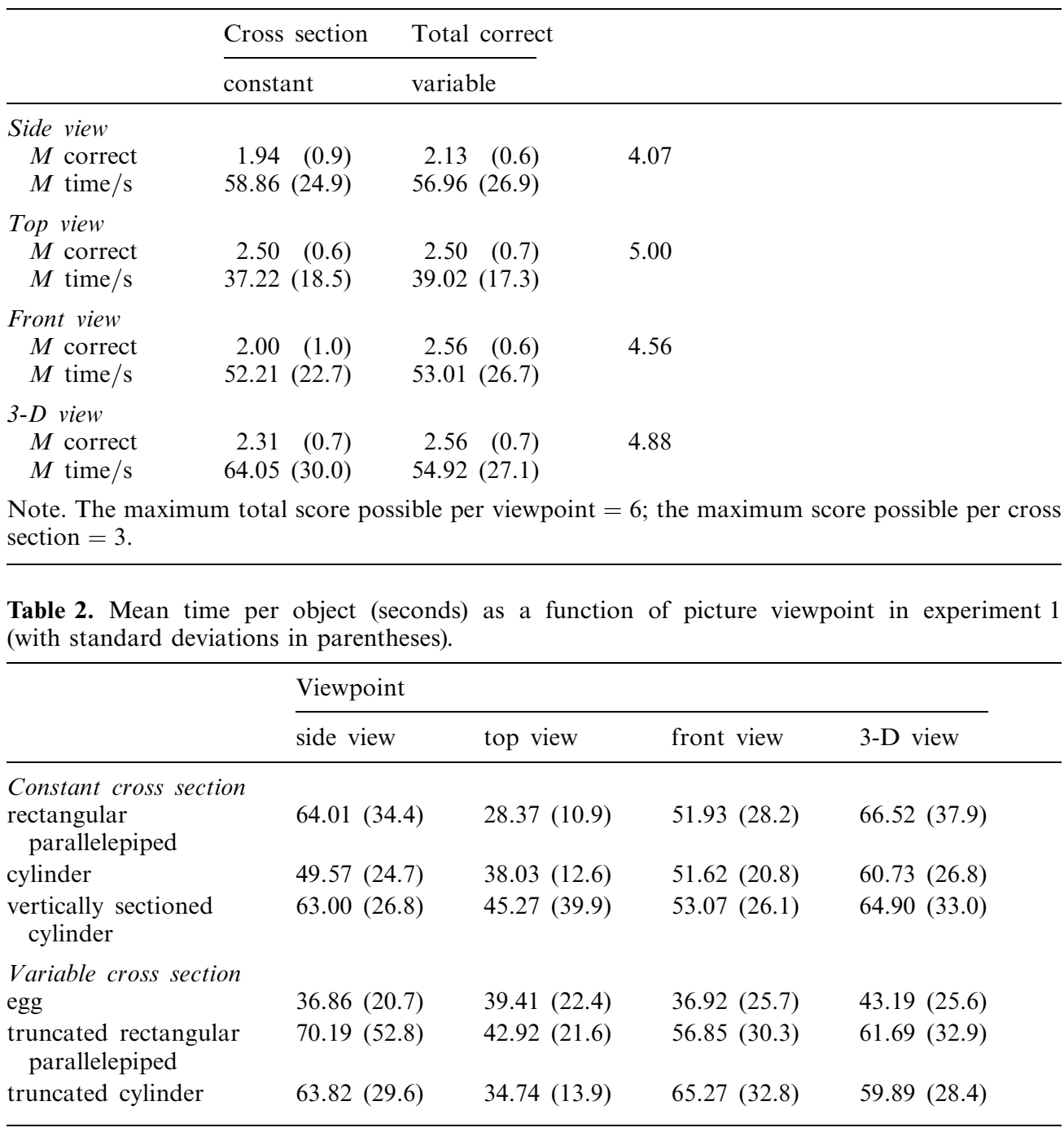

\section{Experiment 2: Viewpoint effects in the blind}

Viewpoint effects in blind subjects were studied in experiment 2 with a novel viewpoint added to earlier research with blind subjects (Heller et al 2002). In addition to frontal, top, and 3-D views, an additional set of pictures involved left side views of the solid objects. Also, this experiment controlled for the variability or lack thereof of the cross section of the stimuli. This manipulation was adopted, since the earlier research by Heller et al did not control for this variable, and the most difficult object in that study was variable in cross section (a pyramid). Consequently, half of the solid stimuli in experiment 2 were of constant cross-section and half were variable. The stimuli with constant cross sections in the vertical axis were expected to yield pictures that would be easier for the subjects to understand, and should have produced increased accuracy scores and reduced response latencies. 


\subsection{Method}

3.1.1 Participants. There were three groups of visually impaired participants each of ten subjects: LB (four females, six males; mean age $=44.4$ years, age range $=26-70$ years), EB (six females, four males; mean age $=52.0$ years, age range $=32-65$ years), and VLV (six females, four males; mean age $=39.6$ years, age range $=22-61$ years), and a group of ten naive BS subjects (mean age $=40.7$ years, age range $=21-76$ years). Detailed information about causes of blindness and age of onset can be found in table 3. The EB subjects were blind at birth, or lost their sight during the first year of life (with the exception of one subject who lost her sight before the age of 2 years).

Table 3. Characteristics of the blind subjects in the haptic viewpoint experiments, including gender, age, education, age of onset, cause of blindness, and presence of light perception.

\begin{tabular}{ccllll}
\hline Gender & Age $/ \mathrm{y}$ & Education & Age of onset/y & Cause & Light per \\
\hline $\begin{array}{c}\text { Early } \\
\text { blind, } E B\end{array}$ & & & & \\
F & 32 & HS & 0 & ROP & no \\
F & 62 & SC & 2 & meningitis & minimal \\
M & 50 & MA & 0 & ROP & yes \\
F & 57 & HS & 0 & RP & minimal \\
F & 33 & JD & 0 & RP & yes \\
M & 51 & JD & 0 & ROP & no \\
M & 52 & HS & 0 & ROP & no \\
M & 60 & BA & 0 & ROP & no \\
F & 58 & BA & 0 & ROP & no \\
F & 65 & BA & 0 & genetic disorder & yes
\end{tabular}

Late blind, $L B$

$\begin{array}{llll}\text { M } & 35 & \text { SG } & 9 \\ \text { M } & 58 & \text { PhD } & 40 \\ \text { M } & 60 & \text { MA } & 3 \text { or } 4 \\ \text { F } & 26 & \text { BA } & 6 \\ & & & \\ \text { M } & 33 & \text { SC } & 23 \\ \text { F } & 39 & \text { SC } & 31 \\ \text { M } & 32 & \text { SC } & 25 \\ \text { M } & 41 & \text { HS } & 13 \\ \text { F } & 70 & \text { BA } & 17 \\ & & & 14\end{array}$

Very low vision, $V L V$

\begin{tabular}{|c|c|c|c|c|c|}
\hline $\mathrm{F}$ & 61 & SG & 11 & retinal degeneration & yes \\
\hline M & 28 & MS & 13 & $\begin{array}{l}\text { retinal degeneration } \\
\text { (LV at birth) }\end{array}$ & yes \\
\hline $\mathrm{F}$ & 42 & MA & 36 & $\mathrm{RP}$ & yes \\
\hline M & 36 & $\mathrm{SC}$ & 32 & $\mathrm{RP}$ & yes \\
\hline $\mathrm{F}$ & 29 & MSW & 0 & ROP & yes \\
\hline M & 42 & MS & 25 & RP & yes \\
\hline $\mathrm{F}$ & 51 & $\mathrm{BA}$ & 20 & retinal detachment & yes \\
\hline $\mathrm{F}$ & 38 & $\mathrm{SC}$ & 30 & diabetes, cataracts & yes \\
\hline $\mathrm{F}$ & 47 & $\mathrm{SC}$ & 21 & $\begin{array}{l}\text { Labers Amerosis } \\
\text { (LV at birth) }\end{array}$ & yes \\
\hline M & 22 & $\mathrm{SC}$ & 0 & Microphthamia & yes \\
\hline
\end{tabular}

Notes. F, female; M, male; LV, low vision; ROP, retinopathy of prematurity; RP, retinitis pigmentosa; SC, some college; SG, some graduate school; BA, college degree; MA, Master of Arts degree; HS, high school diploma; PhD, Doctor of Philosophy degree; JD, law degree; MSW, Master of Social Work. 
The VLV subjects were all Braille readers and long-cane users. Most could not see hand motion, but two said that they could. The two subjects with sight of close hand motion had vision occluded during the research: one kept his eyes closed, and the other wore a blindfold. The VLV participants had little more than minimal light perception, but a few had large-object perception. The subjects with large-object perception could see the crude shape of a large window if it were very brightly lighted, or the horizon on a bright day. The VLV subjects had diffuse light perception and had the ability to locate very strong light sources. The LB subjects either had no light perception, or, if they did, they lacked the ability to localize light sources, and were unable to see any shapes or close hand motion.

The subjects were recruited in Illinois, from the areas of Bloomington, Charleston, Champaign/Urbana, Homer, Springfield, and Crystal Lake. More than half of the visually impaired subjects were naive to research on viewpoint or perspective, but some served in a previous study more than 3 years ago (Heller et al 2002). Note that all of the standard stimuli and pictures were different in the earlier report (Heller et al 2002).

3.1.2 Stimuli and apparatus. The stimuli and apparatus were identical to those used in experiment 1 , with the sole exception that the black tangible lines were painted an off-white with acrylic paint. Painting the lines reduced their contrast and visibility to render them invisible to persons with a severe visual handicap. This modification of the stimuli was used to offer an additional control to insure that the VLV subjects could not localize the patterns in space.

3.1.3 Design and procedure. The experiment was a between - within design, with independent groups for visual status (EB, LB, VLV, and BS), with repeated measures on viewpoint (front, left side, top, 3-D), and cross section of the stimuli (constant and variable). In most respects, the procedure was identical to that of the first experiment. Viewpoint was blocked, but the viewpoints and pictures were presented in random sequences. As in experiment 1 , the viewpoint of the choice set was explained to each subject prior to each block of trials.

More than half of the visually impaired subjects were completely naive about tangible pictures. To familiarize them with tangible depiction, they were asked to make raisedline pictures of each of the target objects prior to the start of the experiment. Subjects were told that the objects they felt would be the ones that they would be tested on in this experiment. They were asked to try to draw the pictures to show that they "were solid' or 'three-dimensional'. This familiarization phase was adopted to reduce the probability of 'subject loss'. Specifically, some older CB persons have been convinced by their teachers that pictures are only suitable for sighted persons. The familiarization procedure was intended to put these individuals at their ease (see Heller 2000).

After the subjects completed the tasks in experiment 2, they also participated in the mental-rotation conditions of the third experiment. It is possible that there could be some learning effects, but the use of a balanced order of presentation of conditions was not adopted for a few reasons. It was thought that starting with the mental-rotation task could have seemed overly difficult to the EB subjects, and this negative bias could have artificially suppressed performance. Some of the EB subjects were convinced that pictures were not suitable or comprehensible for blind people (see Heller 2000). It was thought that starting with the standard task in experiment 2 would be less likely to yield negative expectancy effects.

\subsection{Results and discussion}

The results are summarized in table 4. An ANOVA was conducted on number correct as a function of visual status (EB, LB, VLV, BS), picture viewpoint (top, front, 3-D, and left side), and cross section of the objects (constant versus variable). 
Table 4. Mean number correct and mean time per object (seconds) in experiment 2 as a function of viewpoint and visual status.

\begin{tabular}{|c|c|c|c|c|c|}
\hline & Viewp & & & & \\
\hline & side & top & front & $3-\mathrm{D}$ & total \\
\hline Late blind, L & & & & & \\
\hline$M$ correct & 4.7 & 5.3 & 4.7 & 5.3 & 20 \\
\hline$M$ time $/ \mathrm{s}$ & 25.50 & 18.20 & 25.43 & 26.42 & \\
\hline Early blind, & & & & & \\
\hline$M$ correct & 3.1 & 4.9 & 3.7 & 4.1 & 15.8 \\
\hline$M$ time $/ \mathrm{s}$ & 34.17 & 24.46 & 40.90 & 38.41 & \\
\hline Very low visi & & & & & \\
\hline$M$ correct & 3.8 & 5.2 & 4.3 & 4.9 & 18.2 \\
\hline$M$ time $/ \mathrm{s}$ & 37.44 & 22.66 & 30.66 & 28.10 & \\
\hline Blindfolded s & & & & & \\
\hline$M$ correct & 4.7 & 5.5 & 4.4 & 5.1 & 19.7 \\
\hline$M$ time $/ \mathrm{s}$ & 41.52 & 27.64 & 47.34 & 39.88 & \\
\hline
\end{tabular}

The effect of visual status failed to reach significance $\left(F_{3,36}=2.52, p=0.07\right)$, but the effect of picture viewpoint was highly significant $\left(F_{3,108}=10.92 p<0.001\right)$. Subjects did much better with the top views than the left side views, with intermediate performance for the 3-D views and front views. A Newman-Keuls test on the means showed that the difference between the left side views and front views was nonsignificant $(p>0.05)$, as was the difference between the top views and 3-D views. However, the comparisons between the means for the top views and the side views and front views was significant $(p<0.05)$, as was the advantage of the $3-\mathrm{D}$ views over the side views. Note that the $3-\mathrm{D}$ views are top views plus a front view. This may explain the advantage of the $3-\mathrm{D}$ views over the side views.

Of special interest, the interaction between visual status and viewpoint was nonsignificant $\left(F_{9,108}=0.64, p=0.76\right)$, suggesting that the EB are able to cope with the different viewpoints presented here. The results suggest that EB participants are able to deal with rotations to cardinal orientations, namely the left side views. It is possible to consider the left side views as a mental-rotation problem, although this was not explicitly stated in the instructions to the subjects. Performance for the left side view was comparable to that for the frontal view pictures.

The effect of cross section on accuracy was highly significant $\left(F_{1,36}=30.16\right.$, $p<0.001$ ), owing to higher performance for the objects with variable cross section than constant cross section, but the interaction between cross section and viewpoint was also significant $\left(F_{3,108}=13.85, p<0.001\right)$. Tests of the simple effect of the interaction between viewpoint and cross section showed that viewpoint did not matter for the objects with variable cross sections $\left(F_{3,108}=0.8, p=0.48\right)$. The other interaction effects failed to reach significance (all $p \mathrm{~s}>0.50$ ). Objects with variable cross sections are not necessarily more difficult than those with a constant cross section in the vertical axis. Lower performance that was previously found for the pyramid is probably specific to that form.

While the BS subjects were somewhat slower than the visually impaired participants, an ANOVA on time scores failed to reveal an effect of visual status on response latency $\left(F_{3,36}=2.61, p=0.067\right)$. The mean response latencies for the EB, LB, VLV, and BS were $34.48 \mathrm{~s}, 23.89 \mathrm{~s}, 29.71 \mathrm{~s}$, and $39.09 \mathrm{~s}$, respectively. The effect of viewpoint of the pictures was significant $\left(F_{3,108}=22.45, p<0.001\right)$, as was the effect of object $\left(F_{5,180}=9.98, p<0.001\right)$, but the interaction between viewpoint and visual status was 
also significant $\left(F_{9,108}=2.07, p=0.04\right)$. Overall, a Newman-Keuls test indicated subjects were significantly faster for the top views $(M=23.24 \mathrm{~s})$, and much slower for the other views; the means for the other views did not differ significantly from each other. Tests of simple effects of the interaction between visual status and viewpoint showed that the only significant difference between the groups of subjects was on the front views $(p<0.01)$, while the other simple effects of visual status as a function of viewpoint were all marginal (all $p \mathrm{~s}>0.09$ ). The sighted and EB subjects were especially slow in their judgments involving the front views.

A number of EB subjects stated that they "...did not have a clue" why they were told that the objects were sometimes elevated to eye-height when they were drawn. A couple of them thought that this was a 'funny' thing to say. These comments reflected their lack of anticipation that drawings could vary with object elevation and viewpoint of the sighted observer, and they also represented a naivete about linear perspective. In addition, some blind participants were very unclear about what could be 'seen' from different viewpoints and what could not be seen. Thus, they had trouble differentiating between appropriate drawings of the cylinder and the sectioned cylinder, because they thought that one would be able to see the top of the cylinder, even when the bottom was at eye-height. Similarly, some subjects really did not know what it meant to see the 'front of the object'. They were uncertain about whether a sighted person would be able to see more than just the closest plane or nearest point of an object, such as the sectioned rectangular parallelepiped.

\section{Experiment 3: Rotated objects}

The standard solid objects in the third experiment were rotated $-45^{\circ}$ from their standard positions of the earlier experiments. The visually impaired and blindfolded participants were told that the stimuli were turned counterclockwise, and that they needed to compensate for this when making their matches. They were informed that the pictures were drawn while the objects were in their straight-ahead position. Thus, the participants were instructed to imagine turning the objects in their minds by $+45^{\circ}$ in a clockwise manner before making their matches. If mental rotation and obliques were a special problem for haptics and the EB, then one might expect lower performance for this group of subjects.

\subsection{Method}

4.1.1 Participants. There were forty volunteers in experiment 3. The visually impaired participants were the same individuals from experiment 2. The BS subjects were the same persons who served in the second experiment (but not in experiment 1).

4.1.2 Stimuli and apparatus. The stimuli and apparatus were like those of the first two experiments. However, the standard stimulus wooden objects were always presented at a $-45^{\circ}$ rotation, and could not be moved as they were fixed in place. The apparatus was modified to include a foam board base with a cutout at this angle. The picture viewpoints included top views, 3-D views, and front views.

4.1.3 Design and procedure. The experiment was a between-within design, with the variables being visual status (EB, LB, VLV, and BS) and repeated measures were taken on viewpoint (top, front, 3-D). The viewpoints were blocked, but presented in a random arrangement.

The subjects were told that the stimuli were rotated, and that they needed to compensate for this when making their judgments, otherwise they could choose the wrong pictures. They were given further clarification by taking their hands and showing them the cutout that housed the rotated stimuli. In addition, the sample stimulus was placed at this rotated angle and then turned so that it was straight-ahead. 
The participants had their hands on the sample object during this demonstration. The subjects were told that they needed to do this in their minds, since the drawings were produced while the objects were straight-ahead. As in the earlier experiments, subjects were timed from the moment they touched the standard objects until they made their choices. No feedback on performance was given.

\subsection{Results and discussion}

Table 5 shows mean number correct for the rotated objects, and indicates somewhat lower performance by the EB participants. An ANOVA was performed on number correct for the variables of visual status (EB, LB, VLV, and BS), viewpoint (front, top, 3-D), and cross section (constant versus variable) and yielded a highly significant effect of visual status $\left(F_{3,36}=3.65, p=0.021\right)$, owing to lower mean scores for the EB $(52.6 \%$ correct $)$, compared with the LB $(84.3 \%$ correct), VLV $(80.7 \%$ correct), and BS (84\% correct) subjects. A Newman-Keuls test on the mean number correct indicated that the differences between EB performance and all of the other means were significant $(p<0.05)$, but the other means did not differ from each other $(p>0.05)$. The effect of viewpoint was highly significant $\left(F_{2,72}=8.14, p<0.001\right)$, because of lower performance for the frontal views compared with the top views and the 3-D views. A Newman-Keuls test showed that the difference between the front views and other views was significant $(p<0.05)$, but the top and 3-D means were not significantly different from each other $(p>0.05)$. However, the main effect of cross section was significant, with higher performance for the forms with variable cross section than those with a constant cross section $\left(F_{1,36}=16.71, p<0.001\right)$. The interaction between cross section of the objects and viewpoint was also significant $\left(F_{2,72}=19.67, p<0.001\right)$. The interaction derived from very low mean performance $(M=51 \%$ correct) for the front views of the objects with constant cross sections (the cylinder, rectangular parallelepiped, and vertically sectioned cylinder). All of the other interaction effects failed to reach significance (all $p \mathrm{~s}>0.37$ ).

An ANOVA on time scores considered the variables of visual status, viewpoint of the pictures, and cross section of the objects. The LB subjects were faster than the others $(M=22.38 \mathrm{~s})$. However, the effect of visual status on response latency failed to

Table 5. Mean number correct and mean time (seconds) for $-45^{\circ}$ rotated objects in experiment 3 as a function of viewpoint, cross section of object, and visual status.

\begin{tabular}{|c|c|c|c|c|}
\hline & \multicolumn{4}{|c|}{ Viewpoint } \\
\hline & top & front & $3-\mathrm{D}$ & total \\
\hline \multicolumn{5}{|c|}{ Late blind, $L B$} \\
\hline$M$ correct & 5.2 & 4.6 & 5.4 & 15.2 \\
\hline$M$ time/s & 19.82 & 25.93 & 21.41 & \\
\hline \multicolumn{5}{|c|}{ Early blind, EB } \\
\hline$M$ correct & 4.5 & 3.1 & 3.8 & 11.4 \\
\hline$M$ time $/ \mathrm{s}$ & 27.22 & 35.30 & 32.18 & \\
\hline \multicolumn{5}{|c|}{ Very low vision, $V L V$} \\
\hline$M$ correct & 5.2 & 4.3 & 5.0 & 14.5 \\
\hline$M$ time/s & 23.41 & 32.24 & 19.02 & \\
\hline \multicolumn{5}{|c|}{ Blindfolded sighted, BS } \\
\hline$M$ correct & 4.9 & 4.8 & 5.4 & 15.1 \\
\hline$M$ time $/ \mathrm{s}$ & 29.07 & 39.01 & 36.24 & \\
\hline \multicolumn{5}{|c|}{ Naive blindfolded sighted (without prior experience) } \\
\hline$M$ correct & 4.4 & 3.5 & 3.8 & 11.7 \\
\hline$M$ time/s & 31.82 & 41.31 & 41.21 & \\
\hline
\end{tabular}


reach significance $\left(F_{3,36}=1.55, p=0.22\right)$, but the effect of viewpoint was significant $\left(F_{2,72}=12.9, p<0.001\right)$. A Newman-Keuls test on mean time scores showed that all of the viewpoint means were significantly different from each other $(p<0.05)$. Subjects were fastest with the top views $(M=24.88 \mathrm{~s})$, but somewhat slower with the $3-\mathrm{D}$ views and slowest with the front views.

It is important to mention that while the task was described as a mental-rotation problem to the subjects, mental rotation of an image was not required. The subjects could solve the problem posed by the rotation task by simply adopting an appropriate vantage point for their hand placement, and then consider this as the front of the rotated object. Thus, subjects merely had to bear in mind the nature of the rotation to solve the problem.

It is entirely possible that part of the difficulty for subjects may have consisted of their confusion about the nature of the manipulation of the objects in the rotation. This rotation of the object was clearly demonstrated for the subjects by having them feel a sample while it was being rotated prior to the test trials. However, one comment by an EB subject was informative, and suggested uncertainty due to lack of task familiarity. After the rotation problem was explained, she asked: "Can other blind people do this?" When MAH replied in the affirmative, she said: "If other blind people can do this, then I can do it!" These comments reflected her concern about task difficulty. Perhaps, part of the problem for the subjects lay in reduced practice with pictures of rotated objects, per se, and not with any limitations in their ability to deal with these complex cognitive problems. The difficulty may be specific to the oblique orientation. Further research will be needed to clarify this issue.

It is important to note that all of the subjects in experiment 3 also participated in the second experiment, and had prior experience with the objects and pictures of them from the 'normal' position without rotation of the objects. Thus, the performance levels of the present experiment could reflect this prior exposure and a learning effect. Consequently, an additional group of twelve BS participants was run on the rotation methods of the present experiment, but without any prior experience with the stimuli, as in the second and third experiments reported here. In most respects, the method was similar to that of the rotation experiment. However, prior to testing, the naive blindfolded subjects were haptically exposed to the objects and told that they would be feeling tangible pictures of those objects.

An ANOVA on number correct compared the BS subjects in experiment 3 with the additional twelve (seven females, five males) naive BS subjects without prior experience with the objects and pictures. Prior experience with the pictures and objects in experiment 2 appeared to have aided subjects during the rotation experiment. Mean number correct was greater for subjects with prior experience than subjects without this prior exposure to the stimuli in experiment 3. The effect of prior experience was highly significant $\left(F_{1,20}=12.66, p<0.01\right)$. The main effect of viewpoint failed to reach significance $\left(F_{2,40}=1.2, p>0.30\right)$, as did the interaction between viewpoint and experience $\left(F_{2,40}=1.47, p=0.24\right)$. Also, the overall mean performance of the naive BS subjects was almost identical to that of the EB subjects in experiment 3 (see table 5).

A second ANOVA on time scores compared the same two groups and considered the variables of viewpoint, experience, and object. The subjects with prior experience were slightly faster $(M=34.77 \mathrm{~s})$ than subjects without this experience $(M=38.1 \mathrm{~s})$, but the main effect of the group comparison failed to reach significance $\left(F_{1,20}=0.28, p=0.60\right)$. The main effect of picture viewpoint was significant $\left(F_{2,40}=11.7, p<0.001\right)$, with faster overall judgments for the top views than the front views or the 3-D views. A NewmanKeuls test indicated that the front views were significantly faster than the others $(p<0.05)$, but the front and 3-D views were not significantly different $(p>0.05)$. 
The rotation problem posed by the present experiment highlighted the difficulty that $\mathrm{CB}$ people may sometimes have in picture-perception tasks. This issue will be considered again in the discussion that follows. EB persons are relatively inexperienced in the interpretation of viewpoints in pictures. Part of their difficulty could derive from a lack of knowledge about what particular viewpoints can inform about objects, rather than any intrinsic problems with image manipulation, per se. Another component of task difficulty could derive from insufficient knowledge about what sighted persons can see when they view objects.

\section{Experiment 4: Picture choices in the vertical/frontal plane}

One reviewer suggested that the results of the first three experiments could be tasklimited, and the advantage of top views may not generalize to other measures of haptic picture perception. Thus, the task arrangement favored top views, since participants could engage in virtually identical tracing motions of the objects and the choice pictures with two hands. This reviewer suggested orienting the choice pictures in the frontal plane as a test of this task-dependence. If top views are really preferred views, then one might still see an advantage for top views.

\subsection{Method}

5.1.1 Participants. The participants were twelve (eight female, four male) naive undergraduate volunteers at Eastern Illinois University.

5.1.2 Stimuli and apparatus. The stimuli were identical to those of experiments $1-3$. However, the target objects were placed on top of an elevated shelf, and the picture choices were mounted on a clipboard attached to the front surface of this shelf (see figure 4).

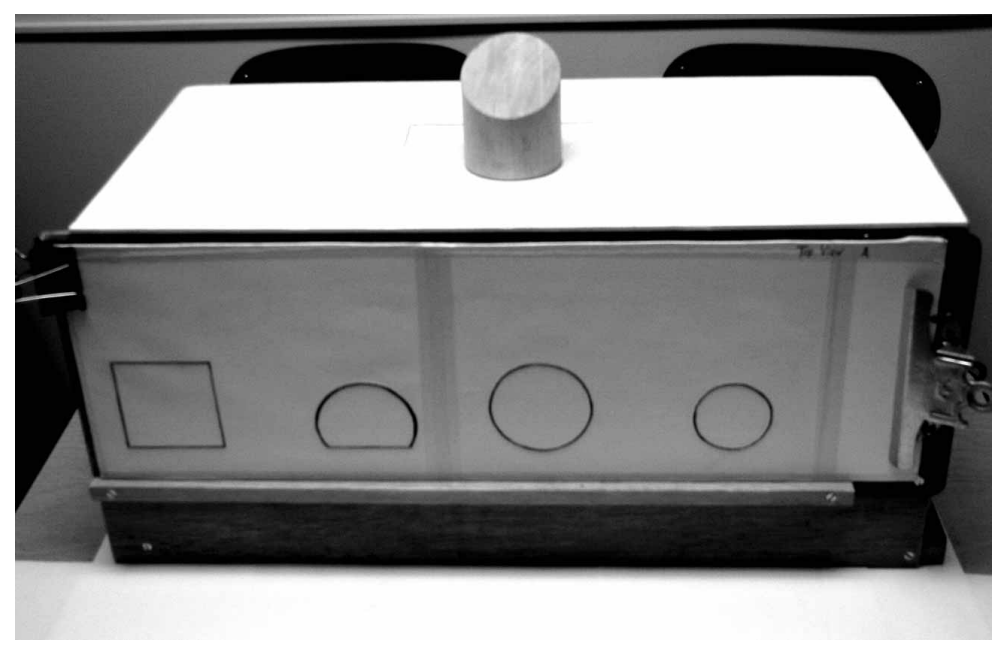

Figure 4. The experimental arrangement used in experiment 4 to place the pictures in the frontal plane. The wooden objects were placed on the top shelf and the haptic pictures were in the frontal plane, as on a CRT display.

5.1.3 Design and procedure. The experiment was a repeated-measures ANOVA, with the variable being viewpoint of the matching pictures (frontal view, left side view, top view, and 3-D viewpoint). Each subject experienced a random order of presentation of the picture viewpoints, but viewpoint was blocked. Each object was presented on the surface of the shelf, and BS participants were instructed to examine all four picture choices before indicating a match. Other than the frontal/vertical orientation of the tangible pictures, the procedure was like that of the first two experiments reported here. 


\subsection{Results and discussion}

In order, mean number correct for the top views, left side views, frontal views, and 3-D views were 5.1, 4.4, 4.2, and 4.8. The effect of viewpoint failed to reach significance $\left(F_{3,33}=2.0, p=0.13\right)$. While top views were slightly easier to identify than other viewpoints, the frontal placement did not appear to modify the effect of viewpoint on accuracy. Mean number correct overall $(M=4.63)$ was identical in experiment 1 and the present experiment, and performance on top views was also similar for the flat placement $(M=5.0$ correct $)$ and the frontal placement $(M=5.1$ correct $)$. However, a second ANOVA on time scores showed that top views $(M=43.5 \mathrm{~s})$ were matched much faster than the other viewpoints. An ANOVA on time scores indicated that there was a significant effect of viewpoint $\left(F_{3,33}=5.0, p<0.01\right)$, a significant effect of object $\left(F_{5,55}=4.6, p<0.01\right)$, and a significant interaction between viewpoint and object $\left(F_{15,165}=1.96, p=0.021\right)$. A Newman-Keuls test on the means showed that the top views were significantly faster than the other views $(p<0.05)$, but the other means did not differ from each other $(p>0.05)$. Tests of the simple effects of the interaction between object and viewpoint indicated that object did not matter for 3-D views. These 3 -D views may provide most useful information about objects, since they include both top and frontal views. This possibility was explicitly tested in experiment 5 .

\section{Experiment 5: Tangible pictures as targets and pictures as choices}

The participants felt tangible pictures and then matched them against sets of picture choices. If pictures convey useful information about objects, then participants were expected to be able to succeed at the matching task. If some viewpoints of pictures are more useful, then one might expect higher performance levels for these views. Given the results of earlier research (eg Heller et al 2002), performance was expected to excel with top views. One might also expect better performance if the target pictures and choices were drawn from the same viewpoint.

Also, the present experiment was intended to determine if the prior speed advantage of top views was task-specific and limited to the particular matching paradigm that was adopted in the earlier experiments reported here. This matching method allowed subjects to engage in identical tracing motions with two hands as they felt the tops of the target objects and top viewpoint choices. One might expect that this could provide an undue advantage for top views, and may not generalize to a more realistic method of appraising possible viewpoint effects in haptics.

\subsection{Method}

6.1.1 Participants. Participants were forty-eight undergraduates (twelve per group) at Eastern Illinois University. There were seven females and five males in each of the groups. All of the subjects were naive to experiments on haptic picture perception.

6.1.2 Stimuli. The stimuli were raised line drawings produced on a Swedish raised-line drawing kit (see figure 4). The Swedish drawing kit produces a durable tangible line when a ball-point pen is drawn over the surface. This drawing kit can be obtained from the Swedish agency for special education (SIH, Laromedel). The pictures depicted a cube, hexagonal prism, triangular prism, rhombic prism, and pyramid. The object models were approximately $8.90 \mathrm{~cm}$ in size, and the pictures were approximately life-sized.

The depictions were drawn from the vantage point of top views, 3-D views, frontal views in parallel perspective without converging lines, and frontal views with converging lines (see figure 5). The stimuli were the same as in earlier research and additional details about the drawings can be obtained from Heller et al (2002).

6.1.3 Design and procedure. The experiment was a between-within design, with independent groups for viewpoint of the target picture (frontal, frontal with converging 

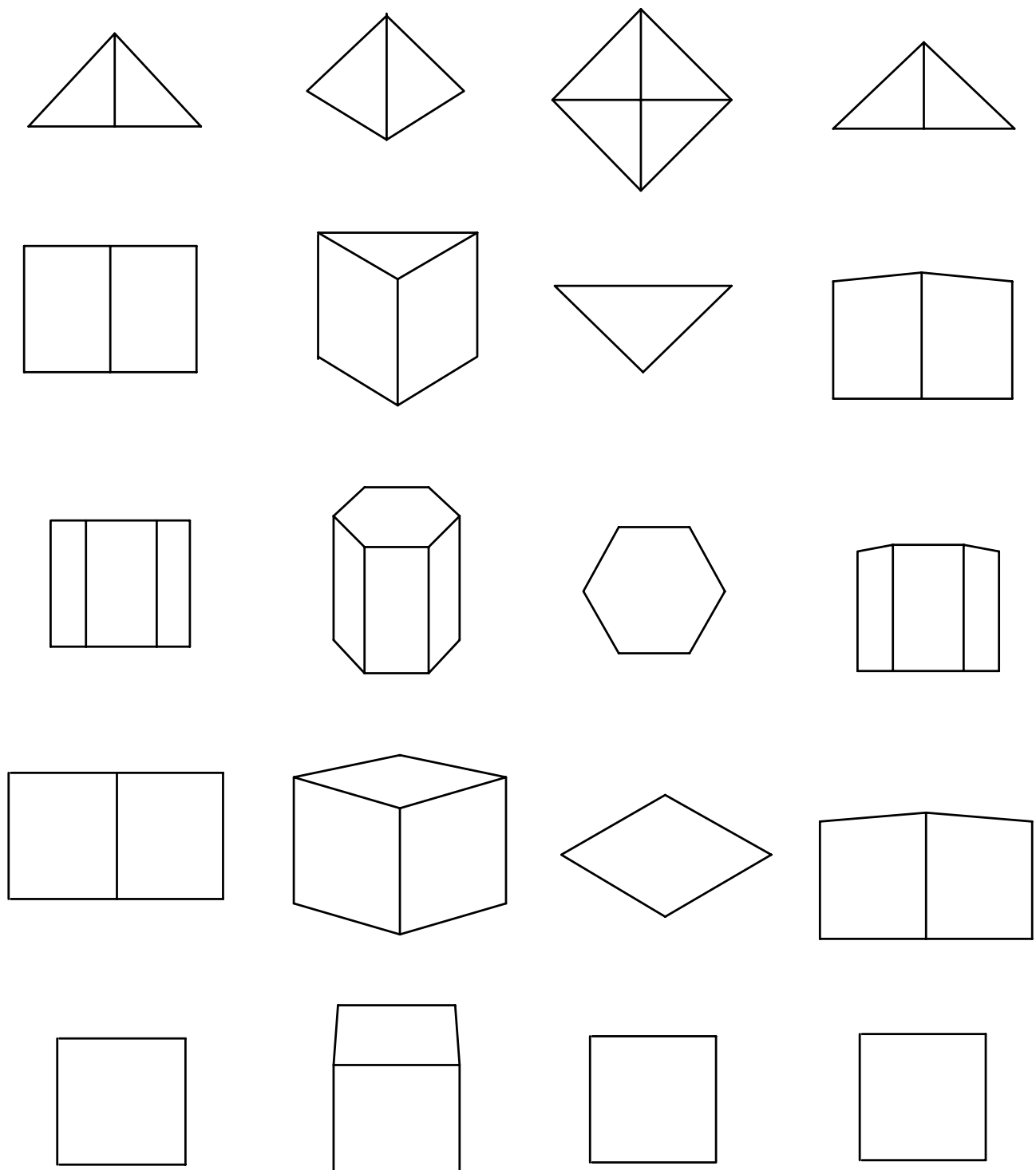

(a)

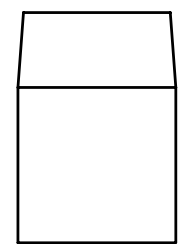

(b)

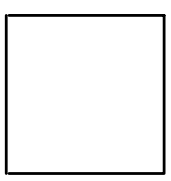

(c)

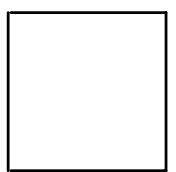

(d)

Figure 5. Pictures used in experiment 5, with pictures as targets and pictures as choices: (a) front view, (b) 3-D view, (c) top view, and (d) foreshortened front view.

lines, 3-D, and top views). Repeated measures were taken on viewpoint of the choice set (frontal, frontal with converging lines, 3-D, and top views). Each subject was exposed to all viewpoints for the picture choices, but the viewpoints were blocked and presented in a randomized order.

Participants were told that they would feel a target picture, and then would feel four picture choices. The participants were instructed that the pictures were drawings of geometrical objects, and that most of them (but not all) had a constant cross section from the top to the base. Subjects never actually felt the objects themselves. They were to feel the picture target and then all four picture choices before indicating which picture showed the same object that was depicted by the target. Subjects were informed about the viewpoints of the pictures for the target and for the picture choice sets. The subjects were told that they would be timed from the time that they first touched the target 
picture until they indicated a match, but they were to try for accuracy. The subjects were permitted to use one or both hands to feel the targets and the choice sets, and were also told that they could go back and forth to examine the target if they wished. Feedback on performance was not given.

\subsection{Results and discussion}

Performance was higher for 3-D viewpoints than all of the other views (see table 6). In order, mean percentage correct for the top, frontal views, frontal views with converging lines, and $3-\mathrm{D}$ views were $57 \%, 58 \%, 60 \%$, and $74 \%$, respectively. An ANOVA on number correct showed highly significant effects of target viewpoint $\left(F_{3,44}=4.23\right.$, $p=0.01)$, but a nonsignificant effect of the viewpoint of the matching pictures $\left(F_{3,132}=0.8, p=0.47\right)$. However, the interaction effect between target viewpoint and choice viewpoint was highly significant $\left(F_{9,132}=10.9, p<0.001\right)$. Tests of the simple effects of this interaction showed that all simple effects were significant (all $p \mathrm{~s}<0.025$ ). The interaction reflected superior performance when the target and matching views were identical. Even when both views were identical, 3-D performance was considerably better than top-view matching accuracy.

Table 6. Haptic pictures as targets and haptic pictures for choices. Mean number correct and standard deviations (in parentheses) for the target viewpoint as a function of choice set viewpoint.

\begin{tabular}{|c|c|c|c|c|c|}
\hline & \multicolumn{5}{|c|}{ Choice viewpoint } \\
\hline & $\begin{array}{l}\text { front parallel } \\
\text { projection }\end{array}$ & $\begin{array}{l}\text { front with } \\
\text { convergence }\end{array}$ & $3-\mathrm{D}$ & top & total \\
\hline \multicolumn{6}{|l|}{ Target viewpoint } \\
\hline $\begin{array}{l}\text { front parallel } \\
\text { projection }\end{array}$ & $3.7(1.0)$ & $3.5(1.1)$ & $3.0(1.1)$ & $1.0(1.0)$ & $12.1(1.9)$ \\
\hline $\begin{array}{l}\text { front with } \\
\text { convergence }\end{array}$ & $3.4(1.2)$ & $3.3(1.6)$ & $2.8(0.8)$ & $2.1(1.2)$ & $11.7(3.6)$ \\
\hline $3-\mathrm{D}$ & $3.3(1.1)$ & $3.2(1.3)$ & $4.8(0.4)$ & $3.6(1.1)$ & $14.8(2.6)$ \\
\hline Top & $2.2(1.1)$ & $2.3(0.9)$ & $2.6(1.0)$ & $4.4(0.8)$ & $11.5(2.1)$ \\
\hline Object as target & $3.4(1.6)$ & $3.5(1.2)$ & $3.5(1.1)$ & $4.8(0.6)$ & $15.3(3.1)$ \\
\hline
\end{tabular}

Subjects were slightly slower when the target was a 3-D picture. An ANOVA on time scores revealed a nonsignificant effect of target viewpoint $\left(F_{3,44}=0.6, p=0.6\right)$, but a significant effect of the viewpoint of the matching pictures $\left(F_{3,132}=6.4, p<0.001\right)$. Participants were somewhat slower for the $3-\mathrm{D}$ view choices $(M=83.3 \mathrm{~s})$ than the topview choices $(M=65.6 \mathrm{~s})$. Subjects may have been slower with 3-D views because they contain more information. However, the interaction between target viewpoint and choice viewpoint was also significant $\left(F_{9,132}=3.0, p<0.01\right)$. This reflected faster responses when the viewpoint of the target and the choice set were the same.

In order to provide a frame of reference to interpret the performance levels found with pictures as targets, an additional comparison group of BS subjects was tested with the wooden objects as targets and the same method as experiment 5. Each of the twelve participants (seven females, five males) was tested with the wooden objects as targets and repeated measures were taken on the four viewpoints. The results were compared with the data from the four groups of experiment 4 (see table 6). The effect of type of target was highly significant $\left(F_{4,55}=5.3, p=0.001\right)$, the effect of picture-choice viewpoint was nonsignificant $\left(F_{3,165}=0.85, p=0.47\right)$, but the interaction between target and choice viewpoint was also highly significant $\left(F_{12,165}=9.5, p<0.001\right)$. 
Performance with the object as target ( $M=15.3$ correct overall) was similar overall to that for a 3-D picture as a target. A Newman-Keuls test comparing the mean number correct for the target viewpoints and wooden objects showed that the 3-D picture and means for wooden objects did not differ from each other $(p>0.05)$, but both were significantly different from the other viewpoints $(p<0.05)$. This indicates that pictures may provide useful spatial information that may sometimes rival performance derived from the solid objects themselves.

The task of feeling picture targets and picture choices was described as very difficult by the sighted controls. It required them to imagine a real object that they never experienced in the experimental setting, and then interpret perspective information that was present in pictures. Note that the 3-D pictures contain top and front views of the objects, and this clearly provides very useful information about the solids that are depicted. Of course, it is not clear if the advantage of 3-D views as targets would also be found with $\mathrm{CB}$ participants, but that is properly the subject of future research. Note that, in the third experiment, 3-D and top views yielded comparable mean accuracy scores. Moreover, the EB participants in experiment 2 did slightly better with $3-\mathrm{D}$ views than with frontal views.

One interpretation of these results may be that the data from experiments $1-3$ indicate that the advantage of the top view is task-specific. However, it bears mentioning that an advantage for top views over other views in a similar matching method (with objects as targets) was also found when subjects were prevented from feeling the tops of the standard objects (see Heller et al 2002). The present results are certainly consistent with this interpretation. However, it is also likely that the 3-D views simply provide more information about the objects that were used as models for the pictures, hence their superiority over top views in this experiment.

\section{Experiment 6: Complex objects as targets}

It was considered possible that the results of experiments $1-3$ could have been limited to the specific stimuli of those experiments. Thus, the results may not generalize to more complex objects. If so, it would be a mistake to generalize about view-dependence from a rather limited set of simple geometrical solids. Thus, a top view of a person's head is not likely to serve as a preferred view and recognition from this vantage point may be very limited. Consequently, in experiment 6 we used more complex wooden objects as targets in a matching task.

\subsection{Method}

7.1.1 Participants. The participants were ten (six females, four males) BS undergraduates from Eastern Illinois University. None served in prior research on haptics.

7.1.2 Stimuli. The stimuli are shown in figure 6, and the tangible matching drawings are presented in figure 7. The viewpoints included front views, top views, and 3-D views. The drawings were produced on swell paper, as in experiments $1-3$. The stimuli were hardwood objects finished with polyurethane varnish, including a lighthouse, snowman, ball with egg, finial, large-medium-small cube (in size order, bottom up), large-smallmedium cube. The objects ranged in size from $14.4 \mathrm{~cm}$ in height (lighthouse) to $10.8 \mathrm{~cm}$ high (large-medium-small cube).

7.1.3 Design and procedure. The experiment was a one-way ANOVA, with repeated measures on viewpoint (top, frontal with converging lines, and 3-D). In most respects, the procedure was similar to that of experiment 1. Participants felt a target object and then felt four picture choices before indicating a match. Each subject was exposed to the six objects in random order, blocked by viewpoint. The order of presentation of viewpoints of the matching pictures was randomized. Participants were not allowed to move the target objects. As in the earlier experiments, feedback on performance was not given. 


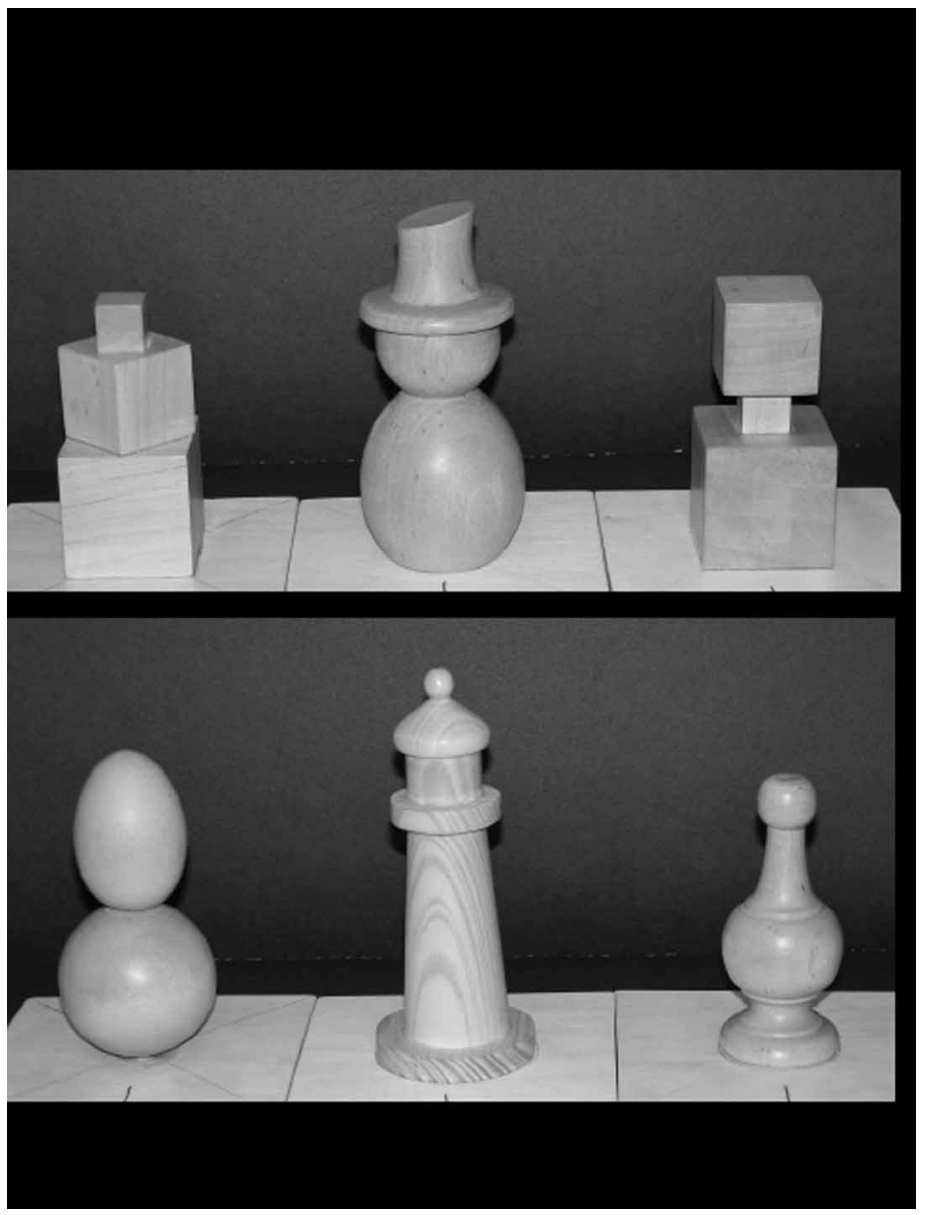

Figure 6. Complex wooden objects from experiment 6.

\subsection{Results and discussion}

Performance was higher for the 3-D viewpoints $(M=5.0$ correct out of 6$)$, with somewhat lower performance for the frontal views $(M=4.8$ correct) and the top views $(M=4.7$ correct). However, an ANOVA on number correct showed a nonsignificant effect of viewpoint $\left(F_{2,18}=0.24, p=0.79\right)$. Faster response latencies were found for top views $(M=38.3 \mathrm{~s})$ than for front $(M=47.3 \mathrm{~s})$ or 3 -D views $(M=54.9 \mathrm{~s})$. An ANOVA on response latency showed that the effect of viewpoint on response time was significant $\left(F_{2,18}=4.7, p<0.025\right)$. However, a Newman-Keuls test on mean response times indicated that the top views were significantly faster than the 3 -D means $(p<0.05)$, but the other comparisons between the means were not significantly different.

The results of experiment 6 show that the viewpoint effects that were found in experiments $1-3$ are probably limited to the stimuli in those experiments. The objects in the present experiment were more complex, and varied across a number of dimensions. Top views lost their accuracy advantage given these complex objects. Thus, a top view drawing of the snowman did not depict an additional circle for the 'belly', since this feature was not visible when viewed monocularly from above. This was the case, despite the slightly larger diameter of that feature. Also, participants had to discriminate top views of some of the objects in terms of the sizes of the circles that were used to represent salient features from above. This was not an easy task, and recognition of top views was certainly not more facile than the recognition of 3-D views. 

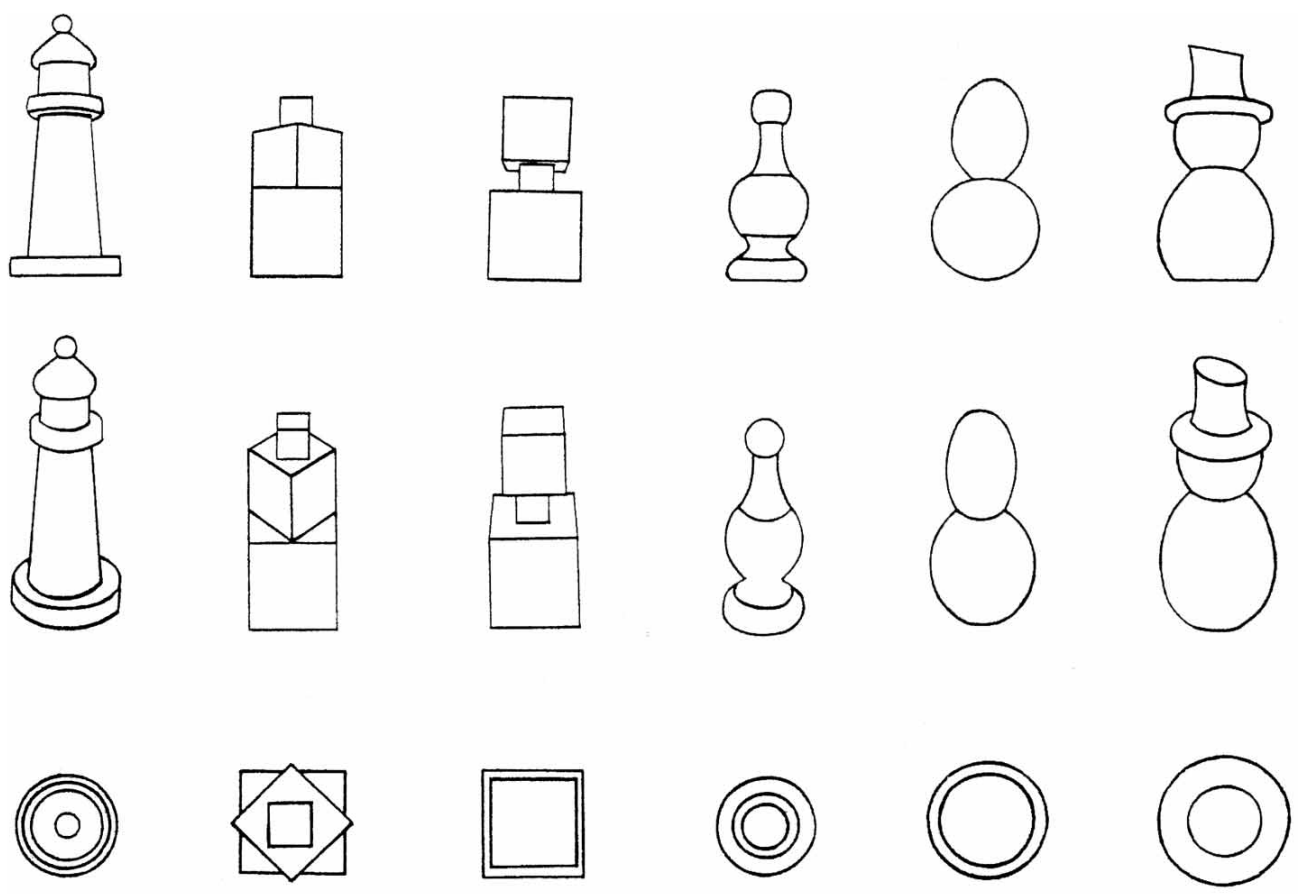

Figure 7. Depictions of complex objects in experiment 6. Front views are at the top of the figure. The middle row shows 3-D views, and top views are at the bottom of the figure.

\section{General discussion}

Performance was high with matching 2-D pictures to solid geometrical objects. As in earlier research, matching top views was fastest for the subjects in experiments $1-4$. Anticipation of side views was especially difficult. The side view required taking an alternative viewpoint (see Heller and Kennedy 1990) and imagining the image that would be projected from that vantage point. 3-D views were easier than side views, but were sometimes more difficult than top views. Accuracy was similar for top and 3-D views in experiment 3. The EB subjects did slightly better with 3-D than with front views in experiment 2. In experiment 5, we used pictures as targets and as choices, revealing an advantage for 3-D views. Moreover, top views did not appear to be privileged in experiment 6, with more complex objects. The results of the experiments, taken together, suggest that viewpoint effects are very much dependent upon stimulus characteristics and task demands.

What do we make of the notion of viewpoint-dependence in touch? The present results suggest that the jury is still out on this question. The method used to evaluate viewpoint effects obviously matters, since 3 -D views were best in experiment 5 . While some might question the idea that $3-\mathrm{D}$ views would be useful for the EB, this result was found in experiment 2 (see table 4). Viewpoint did not have as large an effect in experiment 6 , but some subjects did find the top views to be most difficult. Further research will be needed to gather more evidence on this theoretical issue. Nonetheless, there are many instances where tangible pictures can convey useful information in the complete absence of objects. It is here that 3-D views may be most useful for haptics and blind persons.

The present results are consistent with the theoretical views on perspective and the relationship between haptics and vision that have been advanced by Kennedy and Juricevic (2006). They hold that haptics and sight are similar in many ways, and so it is no wonder 
that blind people make use of aspects of perspective in their depictions (see Kennedy 1993). Heller and Kennedy have argued that vantage points can be adopted by the EB (see Heller and Kennedy 1990), since directions are comprehensible for touch as for vision (Kennedy 1993). Perspective transformations involve distortions induced by modifications in the vantage point of the observer, and should make sense to blind people, as for the sighted, since directions are understood by touch. Many CB persons, however, have not had any experience with drawings, and this contributes to a lack of familiarity with linear perspective. Thus, half of the CB persons in the present study did not automatically understand why a drawing might vary as a function of elevation, and commented on this. Moreover, this lack of familiarity with depiction led these EB persons to express a lack of understanding of what 'can be seen' from the front of an object. Similarly, in earlier research, a CB person did not automatically anticipate the idea that perspective drawings of a square were not square (see Heller et al 2002). Moreover, many CB individuals did not spontaneously include foreshortening in their drawings of a board at a slant (Heller et al 1996). Perspective is a very sophisticated method for depicting spatial arrays, and was invented by Brunelleschi in the Renaissance (Panofsky 1925/1991). Most sighted persons cannot draw in correct perspective, and must be taught to do this. Art instructors will attest to the fact that not all sighted individuals are successful at this.

However, it is clear that blind people may rapidly learn to appreciate perspective information (Heller et al 1996). This suggests that there may be a fundamental similarity between haptics and sight. Both vision and touch respond to contour information in objects, and edges may be felt as well as seen. Lines in visible or tangible form can represent edges, and so one may expect that both senses will respond to this basic attribute of forms, whether in 2-D or 3-D representations.

The results of the present experiments are relevant to some current theoretical formulations that assume that visual imagery and visual experience are necessary for perception of 2-D tangible arrays (eg Lederman et al 1990). Lederman et al suggested that visual mediation was necessary for the interpretation of haptic pictures. Sighted subjects report engaging in visual imagery when touching objects and pictures, but this does not means that visual mediation is a prerequisite for the interpretation of tangible displays. EB subjects were able to make sense of these pictures, although they did have somewhat lower performance in the mental-rotation task of the third experiment. Haptic pictures can yield high levels of performance, and need not suffer when compared with 3-D objects. Note that performance with tangible pictures in experiment 5 often exceeded that reported for matching 3-D faces (Kilgour and Lederman 2002). The complexity of the stimuli obviously matters, but matching accuracy reached $83.3 \%$ correct in experiment 6 with rather complex objects, and $96 \%$ correct in experiment 5 with 3-D pictures as targets and as choices.

There are practical implications of the present results on perspective and viewpoint. Certainly, EB persons may be less experienced with drawings that adopt a particular vantage point, and this should be considered when they are introduced to graphics. Some blind individuals have told MAH that "they know that sighted people see half a tree, but blind people imagine the whole tree". This sort of comment reflects an individual's awareness of viewpoint, and his/her tendency to think of an object in terms of a 3-D solid, and not just from one vantage point. The results of the present experiments suggest that the EB can certainly comprehend pictures from varying viewpoints, but probably require some experience before it is reasonable to expect facility with some complex sorts of perspective transformations. This is suggested by the results of experiment 3 with object rotation.

The lack of visual experience did not lower performance for stimuli in cardinal orientations. However, increases in task difficulty with object rotation yielded lower 
matching accuracy for the EB subjects. This lower performance could reflect the impact of a number of variables, which will be considered in turn.

First, touch may be limited in capability when spatial tasks require active manipulation of spatial imagery (see Cornoldi and Vecchi 2000). Matching side views with the objects in a straight-ahead position requires active visuo-spatial imagery. The EB subjects in experiment 2 had somewhat lower performance for side views, with particularly low accuracy for the stimuli with a constant cross section in the vertical axis (eg cylinder, truncated cylinder, rectangular parallelepiped). It is unlikely, however, that the results of experiment 3 are explainable solely in terms of problems with active visuo-spatial imagery, since the main effect of visual status was nonsignificant, as was the interaction between visual status and viewpoint in experiment 2 . The difficulty could be linked to mental-rotation tasks, since the difference between the EB and the other subjects did not appear to be very large until the stimuli were presented at the $-45^{\circ}$ orientation. This result is consistent with earlier reports of the impact of oblique orientations on haptics, as in Braille (Heller et al 1999). EB subjects in that earlier report had lower performance when the Braille characters were at oblique orientations. Note that EB subjects in the study of Heller et al (1999) had high performance when coping with left - right reversed Braille, and they also were experienced with coping with this mental manipulation. Readers of tangible Braille are likely to have used Braille slates for writing, and these devices require left - right reversals of the stimuli.

Visual experience, or a lack thereof, could influence performance in haptic mentalrotation problems in a number of ways. First, visual imagery may be helpful in coping with obliques. However, sighted subjects showed lower performance when they did not have any prior experience with the stimuli (see experiment 3). This suggests that visual experience may simply speed up the learning process for people familiar with the stimuli that are 'at hand'. The BS participants probably showed higher benefit from their prior experience in experiment 2 than did the EB subjects. This does not mean that EB persons, and the sense of haptics, cannot successfully engage in mental rotation given obliques. It is just possible that they may require more experience to learn to cope with these oblique or varying angular rotations. This is especially likely given very novel stimuli and unfamiliar tasks.

Most of the EB subjects were unfamiliar with drawing tasks, and this lack of experience could certainly have contributed to lower performance in experiment 3 . Note that a number of the EB participants were unclear about what parts of an object could be 'seen' from different vantage points. Thus, one subject asked if front views limited the viewer to sight of just the closest surface plane of the object. For example, some of the solid objects were presented so that the front views included a close planar surface (eg the truncated parallelepiped) and a receding angular plane. The experimenter replied by taking the subject's hand, moving it toward the front of the object, and saying: "Imagine you are looking at the object from this vantage point? What could you feel?" The experimenter made certain that the subject's fingers touched more than just the closest surface during this reply, and asked: "What do you think a sighted person could see from this viewpoint?" If one has never seen, then the viewpoint task involves imagining what a sighted person can see when confronted by solid objects. Touch can experience all parts of an object at once, if the object is small enough. Thus, EB individuals could find the instruction to imagine a front view, or a side view, as inherently ambiguous. They could clearly make sense of the problem, given their relatively good performance overall in experiment 2. Of course, vantage point can matter for touch as for vision if objects are large enough. Thus one may not be able to see all sides of a large object at once. The same holds true for touch and haptic exploration of large objects. Nonetheless, lack of familiarity with the task could have made the EB subjects especially susceptible to any increases in task difficulty, as with the oblique rotations of the objects. 
Part of task difficulty for the EB subjects involved lack of experience with perspective problems. This lack of experience was revealed in the spontaneous comments of the subjects about a lack of understanding why they were being told about the relative height of the objects. However, this difficulty with perspective is probably not insurmountable, given the relative success of the EB participants in experiment 2. It is suggested that experience with perspective, perhaps involving explicit instruction, is likely to yield considerable improvement in this area by EB persons.

Visual imagery may not be all that different from haptic imagery, but it simply could be easier to manipulate visual images. This seems likely, since not all of the EB subjects did poorly on the rotation problem. This suggests a quantitative, rather than a qualitative difference in the efficacy of visual versus haptic imagery.

Acknowledgments. This research was supported by NSF grant BCS-0317293 from the program in Perception, Action and Cognition. We are grateful to Lindsey Mitts for help with running subjects in experiment 1 .

\section{References}

Biederman I, 2001 "Recognizing depth-rotated objects: A review of recent research and theory" Spatial Vision $13241-253$

Biederman I, Gerhardstein P C, 1993 "Recognizing depth-rotated objects: Evidence and conditions for three-dimensional viewpoint invariance" Journal of Experimental Psychology: Human Perception and Performance $19162-182$

Cornoldi C, Vecchi T, 2000 "Mental imagery in blind people: The role of active and passive visuospatial processes", in Touch, Representation and Blindness Ed. M A Heller (Oxford: Oxford University Press) pp $143-181$

Eriksson Y, 1998 Tactile Pictures: Pictorial Representations for the Blind, Doctoral thesis, Goteborg University, Sweden

Heller M A, 1989 "Tactile memory in sighted and blind observers: The influence of orientation and rate of presentation" Perception 18121 - 133

Heller M A, 2000 "Guest editorial: Society, science, and values" Perception 29 757-760

Heller M A, 2002 "Tactile picture perception in sighted and blind people" Behavioral Brain Research $13565-68$

Heller M A, Ballesteros S (Eds), 2006 Touch and Blindness: Psychology and Neuroscience (Mahwah, NJ: Lawrence Erlbaum Associates)

Heller M A, Brackett D D, Scroggs E, Steffen H, Heatherly K, Salik S, 2002 "Tangible pictures: Viewpoint effects and linear perspective in visually impaired people" Perception $31747-769$

Heller M A, Calcaterra J A, Tyler L A, Burson L L, 1996 "Production and interpretation of perspective drawings by blind and sighted people" Perception 25321 - 334

Heller M A, Calcaterra J, Green S, Lima F, 1999 "The effect of orientation on braille recognition in persons who are sighted and blind" Journal of Visual Impairment and Blindness 93 416-419

Heller M A, Kennedy J M, 1990 "Perspective taking, pictures and the blind" Perception \& Psychophysics $\mathbf{4 8} 459-466$

Holmes E, Hughes B, Jansson G, 1998 "Haptic perception of texture gradients" Perception 27 $993-1008$

Kennedy J M, 1993 Drawing and the Blind (New Haven, CT: Yale University Press)

Kennedy J M, 2000 "Recognizing outline pictures via touch: Alignment theory", in Touch, Representation and Blindness Ed. M A Heller (Oxford: Oxford University Press) pp 67-98

Kennedy J M, 2003 "Drawings from Gaia, a blind girl" Perception 32321 - 340

Kennedy J M, Juricevic I, 2006 "Form, projection and pictures for the blind", in Touch and Blindness: Psychology and Neuroscience Eds M A Heller, S Ballesteros (Mahwah, NJ: Lawrence Erlbaum Associates) pp 73-93

Kerr N H, 1983 "The role of vision in 'visual imagery' experiments: Evidence from the congenitally blind" Journal of Experimental Psychology: General 112 265-277

Kilgour A R, Lederman S J, 2002 "Face recognition by hand" Perception \& Psychophysics 64 $339-352$

Lederman S J, Klatzky R L, Chataway C, Summers C D, 1990 "Visual mediation and the haptic recognition of two-dimensional pictures of common objects" Perception \& Psychophysics 47 $54-64$ 
Marmor G S, Zaback L A, 1976 "Mental rotation by the blind: Does mental rotation depend on visual imagery?" Journal of Experimental Psychology: Human Perception and Performance $2515-521$

Millar S, 1994 Understanding and Representing Space: Theory and Evidence from Studies with Blind and Sighted Children (Oxford: Clarendon Press)

Newell F N, Ernst M O, Tjan B S, Bülthoff H H, 2002 "Viewpoint dependence in visual and haptic object recognition" Psychological Science 12 37-42

Panofsky E, 1925/1991 Perspective as Symbolic Form (New York: Zone; original work published in 1925)

Prather S C, Sathian K, 2002 "Mental rotation of tactile stimuli" Cognitive Brain Research 14 $91-98$

Revesz G, 1950 Psychology and Art of the Blind (London: Longmans Green and Co)

Tarr M J, Bülthoff H H, 1995 "Is human object recognition better described by geon structural descriptions or multiple views? Comment on Biederman and Gerhardstein (1993)" Journal of Experimental Psychology: Human Perception and Performance 21 1494-1505

Tarr M J, Williams P, Hayward W G, Gauthier I, 1998 "Three-dimensional object recognition is viewpoint dependent" Nature Neuroscience $1275-277$

Thompson L, Chronicle E, 2002 "Feeling pictures: The roles of pictorial convention and visual experience in haptic recognition", paper presented at the International Conference on Touch, Blindness and Neuroscience Madrid, Spain, October 16

Thompson L J, Chronicle E P, Collins A F, 2003 "The role of pictorial convention in haptic picture perception" Perception $32887-893$

Zimler J, Keenan J M, 1983 "Imagery in the congenitally blind: How visual are visual images?" Journal of Experimental Psychology: Learning, Memory and Cognition 9 269-282 


\section{PERCEPTION}

VOLUME 352006

www.perceptionweb.com

Conditions of use. This article may be downloaded from the Perception website for personal research by members of subscribing organisations. Authors are entitled to distribute their own article (in printed form or by e-mail) to up to 50 people. This PDF may not be placed on any website (or other online distribution system) without permission of the publisher. 